\title{
Oncology nursing supportive care during the COVID-19 pandemic: reality and challenges
}

\author{
Rebecca A. Clark-Snow ${ }^{1} \cdot$ Cynthia Rittenberg $^{2}$ \\ Published online: 8 February 2021 \\ (C) The Author(s), under exclusive licence to Springer-Verlag GmbH, DE part of Springer Nature 2021
}

The social construct of our global society has been significantly impacted by the SARS-CoV-2 Coronavirus (COVID-19) virus. The health risks of SARS-CoV-2 and its associated disease COVID-19 are better understood, and public health agencies, physicians, government, and health advocates continue to facilitate public access to this information. For many countries, the pandemic has resulted in failing economies, with many facing financial hardship, job loss, and nutrition insecurity. Most importantly, healthcare systems worldwide have made significant adjustments in order to care for patients diagnosed with the virus, as well as maintaining health and preventing disease within our worldwide community.

As of December 11, 2020, the Johns Hopkins University of Medicine Coronavirus Resource Center [1] which has been tasked with providing updated daily statistics regarding the international response to COVID-19 reported the following sobering statistics from 191 countries/regions: Global Cases more than 69 million; Global Deaths 1.5 million plus; US Confirmed Cases more than 15 million; US Deaths more than 292,000. The World Health Organization [2], US-based Centers for Disease Control [3], and local public health organizations worldwide have issued guidelines to inform individuals of the signs and symptoms of COVID-19, as well as identify those who are at higher risk for serious illness. Older adults, those with underlying conditions such as heart and lung disease and diabetes, patients with cancer, certain racial and ethnic minority groups, and people with disabilities and developmental and behavior disorders are among those who must take extra precautions to avoid contracting the virus. Responsible government officials have been providing detailed information, guidelines, and resources for all citizens

Rebecca A. Clark-Snow

rclark_snow@yahoo.com

1 Oncology Supportive Care Consultant, Overland Park, KS, USA

2 Rittenberg Consulting, Metairie, LA, USA regarding the COVID-19 virus, as well as sharing new mitigation developments, and making accurate testing available. Equally important is alerting the public with frequent and timely updates of vaccine development, of which 3 have completed clinical trial enrollment to date. Data evaluation, with potential application submissions to the US Food and Drug Administration for emergency use authorization, is in progress for these vaccine candidates. The hope and expectation are that when COVID-19 vaccines become available, their validity is accepted by the general public, and will ultimately be administered worldwide. Only then can we truly anticipate that the pandemic will be controlled and prevented, with society achieving a state of normality.

Patients with cancer who may be receiving a variety of treatments are at a higher risk of developing severe infections and have increased mortality, especially if they are immunocompromised and diagnosed with COVID-19 [4]. Caring for patients during the pandemic has required that changes to every aspect of what constituted standard of care be reevaluated and adapted to accommodate for the specific precautions and guidelines outlined by institutions and public health agencies. Both oncology medical and nursing professional organizations (ASCO, ONS, MASCC/ESMO, ISNCC, AMA, ANA, AACR, NCCN) have published position papers on COVID-19, in addition to a wealth of papers by clinicians that offer analyses of the impact of the virus on patient outcomes, socio-economic and socio-political factors that influence psychological morbidity $[5,6]$, and issues related to cancer survivorship [7]. COVID-19 symptom presentation is quite similar to symptoms experienced by patients with cancer by virtue of diagnosis, or those receiving treatment. It has therefore become increasingly important during patient education sessions with patients and caregivers to review treatment side effects and COVID-19 symptoms, with specific instructions including contact information, as to when to contact either their nurse or physician.

Oncologists have equated clinical decisions regarding treatment options, while taking into account hospital resources 
such as personal protective equipment as ethical challenges that affect not only patients but also caregivers and all healthcare personnel involved in the care of patients in all phases of treatment, and the continuing overall effective day to day management of institutions and private practices. Risks for patients and the safety of practitioners are factored into the decision- making process. Institutions within the USA, and worldwide, have adapted care delivery models to guide cancer practice and deliver optimal patient care during COVID-19 [4], and therefore a reduction of COVID-19 cases.

Telehealth and telemedicine visits not only have become the norm for patients being evaluated and treated for COVID-19 but also have become an option for cancer practices to protect vulnerable patients, and staff. Every aspect of patient care has been adapted to conform to telemedicine's capabilities. Interim physician visits, initial and interim advance practice nurse visits, nurse-led virtual education sessions with patients and caregivers to review scheduled treatment, and coordinating visits with ancillary services, such as social work, are taking place virtually. The continuing care of individuals with cancer has always been dependent on a multidisciplinary and collaborative approach, which has taken on a greater sense of urgency during the pandemic. So, how are oncology nurses providing supportive care for their patients during the pandemic?

My co-author and I extended an invitation to oncology nursing colleagues in the USA, Canada, Africa, Australia, Spain, Sweden, and Turkey, to consider answering 8 questions in an effort to obtain international perspectives on oncology supportive care nursing during the COVID-19 pandemic, which may be shared among oncology nurses and the oncology healthcare community globally. The following are the questions offered for consideration, which were e-mailed in July and August 2020.

1. Many institutions within the US and other countries have incorporated the use of Telehealth/Telemedicine patient visits in oncology and other medical practices. How has telemedicine impacted your oncology nursing practice?

2. Has telemedicine increased your workload? Have you been included in the actual telemedicine visits with the physician, patient and family members?

3. Regarding patient follow-up after treatment, surgery, etc., how has communication between the patient and nurse changed, if at all, during the pandemic?

4. Do you find that you or your staff are attempting to contact/call patients more often, as clinic visits have decreased in some practices during the pandemic?

5. How has patient education and teaching been impacted; i.e., review of treatment plans, distribution of patient education materials, development of new materials that incorporate COVID-19 precautions? Has the teaching been accomplished face to face, by mail, or E-mail?
6. What changes if any have been made to the provision of supportive care to your patients?

7. Is there any advice you'd like to share with our colleagues that might assist and be beneficial with improving supportive care during the pandemic crisis?

8. How do you envision the immediate future of oncology nursing meeting the supportive care needs of our patients?

We also felt it was necessary to acknowledge the emotional and physical toll the pandemic is having on our nursing community, in fact on all essential personnel and healthcare workers, and asked that our respondents feel free to comment on this most important aspect if they cared to. The questionnaire was e-mailed to 14 nurses, and we received responses from 7 nurses from the USA, Australia, Spain, and Sweden. We are grateful that our colleagues were able to provide the following valuable insight, while managing busy practices.

Supportive care of patients with cancer is multifaceted and encompasses the prevention, management, and alleviation of symptoms of cancer treatment, whether physical or psychological, across the entire cancer continuum, including family members and caregivers (www.MASCC.org). Oncology nurses have developed and use evidence-based guidelines relevant to nursing practice, and have collaborated on the development of evidenced-based guidelines to prevent treatmentrelated side effects in both adults and pediatric patients. With the onset and continuation of the pandemic, oncology nurses have continued to provide patients with the education, communication, tools, and compassionate care to successfully navigate through cancer diagnoses and treatment. However, specific limitations regarding patient contact and follow-up have resulted from the gravity of this insidious virus. Several of the questionnaire respondents already had telemedicine in place in their institutions, initially used as a remote healthcare model, ultimately becoming part of common practice. For others, bringing telemedicine on board came with specific challenges, including device availability and lack of knowledge by some patients of the technology to connect provider and patient. Some patients expressed a preference for scheduling clinic visits with a healthcare provider and were more comfortable with this option rather than a remote telemedicine encounter. Stress, anxiety, and psychological and emotional pressure among patients and staff were not uncommon for some, as telemedicine in some instances increased an already busy workload. Important information discussed during a telemedicine visit was reported by some nurses as not being retained by patients, prompting multiple phone calls to review patient concerns; to address supportive care needs, treatment options, and pre-treatment education; and to schedule appointments. An advantage and benefit for the use of telemedicine noted by our colleagues are that family members unable to accompany patients to clinic/office visits can now be present to support patients during this visit, and be an additional 
resource to review important information after the visit, and provide additional pertinent observations not recalled by the patient. Barriers to the optimal use of telemedicine include the challenge some older patients face with using digital tools; those with limited economic resources may not obtain the care needed, with some providers preferring face to face consultations and office visits. Once again, if the appropriate technology was not available in patient's homes, only phone calls were made by physicians and nurses.

Additional feedback from our colleagues revealed that for many patients scheduled to receive, for example, several cycles of an oral treatment, patients informed their nurses they were satisfied with receiving nurse-initiated phone calls at home to discuss potential side effects prior to starting a new treatment, rather than visiting the hospital quite so often. Follow-up calls to patients to assess and evaluate side effects such as hand-foot syndrome can be problematic; therefore, telephone visits are best reserved for patients who tolerate treatment well and do not require frequent physical visits.

The impact of COVID-19 on patient education was similar in all countries. COVID-19 precautions (temperature check, facemask requirement, quarantining, symptoms to report) were all reviewed with patients prior to initial and follow-up clinic visits via mailed instructions, phone calls from nursing reviewed screening questions and the process for the day of visit. For those patients for whom surgery or a procedure has been scheduled, the importance of scheduling a COVID test within the allowed window prior to the procedure has become part of standard planning and education. Medication review is accomplished via mailed material, face to face encounters, Email, and patient portals if available. Group teaching sessions were cancelled due to social distancing. We also asked if there were changes to the supportive care provided to patients in the wake of COVID-19. Notably when stay at home orders were mandated, some supportive care services were unavailable, such as social work, psychologist, genetic consults, and financial advisors. Once the order was rescinded, patients could either schedule an in-person visit or utilize the telehealth option. It was also reported that an institution developed guidelines as to how to prioritize between patients and treatments during the pandemic; however, use of the guidelines was deemed unnecessary, allowing for the continuation of supportive care as usual.

As oncology nurses, who have not previously cared for patients during a global health emergency, guidance from institutions and professional organizations on best practices is reviewed and incorporated into institutional guidelines as needed. Our colleagues have shared the following advice which we may already have been incorporated to improve supportive care at this time: inform patients which services are still available, and what alternatives methods can be pursued if they have been placed on hold during the pandemic; confirm that patients have access to a phone in case of an emergency and for telephone consults; and who they can reach out to for questions or concerns which will provide peace of mind. I believe this following comment was perhaps the most creative and highlights nursing ingenuity: "If you think something might work, ask if you can do it." The consensus is that during these difficult times, as part of a multidisciplinary team approach, we are attempting to meet the supportive care needs of oncology patients as we always have, that supportive care is evolving and needs to be improved to meet complex circumstances.

The COVID-19 pandemic continues to deliver an emotional and physical toll on patients, all healthcare providers, and our global community. Everyone committed to the care of patients with COVID-19; our patients with cancer and other diseases deserve our most sincere gratitude for unselfishly putting their lives on the line every day. Supportive care of patients with cancer during the pandemic has positioned oncology nurses to continually update their knowledge base of the effect COVID-19 is having on cancer treatments and what used to constitute standard of care. Patient education, managing supportive care needs, and providing reassurance and information regarding available resources during the pandemic are all necessary care components offered by dedicated oncology nurses globally.

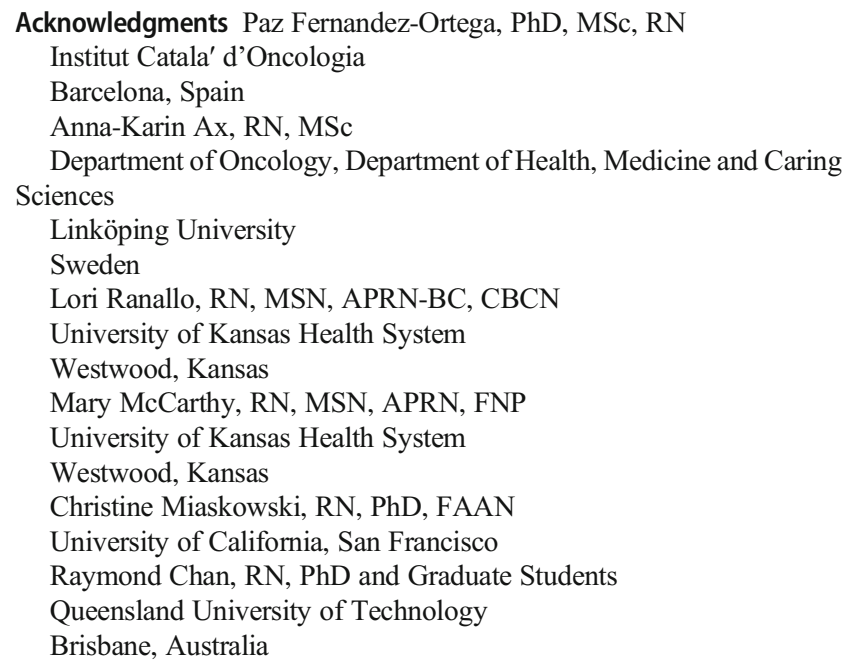

Availability of data and material Material transparency provided by both authors.

Code availability Not applicable

Authors' contributions Both authors contributed equally in the writing of this editorial.

\section{Declarations}

Ethics approval Not applicable

Consent to participate Not applicable 
Consent for publication The authors provide consent for publication.

Conflict of interest The authors declare no competing interests.

\section{References}

1. Johns Hopkins University of Medicine, Coronavirus Resource Center www. Coronavirus.jhu.edu

2. World Health Organization: www. who.int

3. Centers for Disease Control and Prevention: https://www.cdc.gov

4. Brown G, Lutz B (2010) Care delivery models guide cancer practice during COVID-19. https://voice.ons.org/stories/care-deliverymodels-guide-cancer-practice-during-covid-19

5. Chaudry R, Dranitsaris G, Mubashir T, Bartaszko J, Riazi S (2020) A country level analysis measuring the impact of government actions, country preparedness and socioeconomic factors on COVID19 mortality and health outcomes. EClin Med. https://doi.org/10. 1016/j.eclinm.2020.100464

6. Young AM, Ashbury FD, Shapiro L, Scotte' F, Ripamonte CI, Olver IN (2020) Uncertainty upon uncertainty: supportive care for cancer and COVID-19. Support Care Cancer 28:4001-4004
7. Chan A, Ashbury F, Fitch M, Koczwara B, Chan RJ Cancer survivorship care during COVID-19-perspectives and recommendations from the MASCC Survivorship Study Group. Support Care Cancer. https://doi.org/10.1007/s00520-020-05544-4

\section{Websites for COVID-19 Position Papers}

American Society of Clinical Oncology: https://www.asco.org Oncology Nursing Society: https://www.ons.org

International Society of Nurses in Cancer Care: https://www.isncc.org European Society for Medical Oncology: https://www.esmo.org American Medical Association: https://ama-assn.org American Nurses Association: www.nursingworld.org American Association for Cancer Research: https://www.aacr.org National Comprehensive Cancer Network: https://www.nccn.org

Publisher's note Springer Nature remains neutral with regard to jurisdictional claims in published maps and institutional affiliations. 